\title{
1 Rapid evolution slows extinctions in food webs
}

2 Peter C. Zee ${ }^{1,2}$, Casey P. terHorst ${ }^{1}$ and Sebastian J. Schreiber ${ }^{2}$

3

$4 \quad{ }^{1}$ Department of Biology, California State University, Northridge

$5 \quad 2$ Department of Ecology and Evolution, University of California, Davis

6

7 Peter C. Zee (peter.zee@csun.edu) - corresponding author

8 Casey P. terHorst (casey.terhorst@csun.edu)

$9 \quad$ Sebastian J. Schreiber (sschreiber@ucdavis.edu)

10

11 Manuscript type: Article

12 Keywords: eco-evolution; food webs; evolutionary rescue; stability; extinction; trait variation

13

14 Online Appendix A: Effects of species richness

15 Online Appendix B: Results from 'standing variation only' regime

16 Online Appendix C: Cost of trait variation

17

18

19

20 


\section{Abstract}

25 Historically, evolutionary changes have been thought to act on much longer time scales than ecological

26 dynamics. However, a recent body of research has demonstrated that evolution that is rapid enough to

27 dramatically affect ecological dynamics can lead to feedbacks between ecological and evolutionary

28 processes. Thus, to understand the stability of ecological communities, we must also consider

29 evolutionary change in the component species. Here, we use individual-based simulations of a

30 quantitative genetic eco-evolutionary model to describe how trait evolution influences the stability of

31 ecological communities. On short time scales, faster evolutionary rates decreased the probability of

32 species extinctions as populations at low densities were rescued via trait evolution. However, on longer

33 time scales, evolutionary had little effect on the number of extinctions. The extent of short-term

34 evolutionary rescue depended on the source of trait variation; populations with variation generated

35 through mutation experienced more rescue events and were less prone to extinction, relative to

36 populations with only standing trait variation. Trait evolution leading to more rescued populations

37 increased the stability of the community on timescales relevant to conservation. Our work highlights

38 the importance of intraspecific trait variation and the evolutionary mechanisms maintaining this

39 variation for community ecology, as well as management of declining populations in a community

40 context. 


\section{Introduction}

46 While ecology and evolutionary biology each play central roles in our understanding of patterns of

47 biological diversity, many ideas in these fields have developed independently. The time scales at which

48 ecological processes and trait evolution operate have long been thought to be distinct, with ecological

49 changes occurring over significantly shorter periods than evolutionary changes. However, recent work

50 has made great strides in understanding how the conflation of these timescales affects both ecological

51 and evolutionary dynamics. Rapid, contemporary evolution can affect ecological processes, such as

52 population dynamics (e.g., Hairston et al. 2005; Turcotte et al. 2011), species interactions (e.g., Post

53 and Palkovacs 2009; terHorst et al. 2010; Schreiber et al. 2011; Vasseur et al. 2011; Becks et al. 2012;

54 Hiltunen et al. 2014; Patel \& Schreiber 2015), community assembly (Pantel et al. 2015), and

55 ecosystem function (Bassar et al. 2010; Miner et al. 2012; terHorst et al. 2014). In addition to our

56 understanding of ecological processes, rapid evolution can have important conservation implications,

57 as it may lead to evolutionary rescue, whereby a population is able to rebound from low population

58 sizes following a change in the environment through changes in genotype frequencies (Lynch and

59 Lande 1993; Gomulkiewicz and Holt 1995; Gonzalez et al. 2013; Carlson et al. 2014; Orr and

60 Unckless 2014).

61 Rapid evolution and evolutionary rescue require intraspecific trait variation to provide the opportunity

62 for evolution to occur within populations (Bolnick et al. 2011). In a closed population, the amount of

63 variation subject to selection at any given time is a result of the standing genetic variation, with

64 additional mutational input over time (Hermisson and Pennings 2005; Orr and Unckless 2014). If

65 evolutionary change is dependent on waiting for rare mutations to arise, then it may be less likely to

66 affect ecological outcomes over shorter time scales. In contrast, evolution can act on standing trait

67 variation immediately. As such, many experimental eco-evolutionary studies have focused on trait 
evolution on standing variation (e.g., Hendry and Kinnison 1999; Reznick and Ghalambor 2001). Trait variation may also have ecological effects independent of evolutionary change, as more variable interactions can significantly alter the mean strength and outcomes of ecological interactions (Bolnick et al. 2011).

Consumer-resource interactions are a fundamentally important interaction in ecology, as they dictate energy flow through ecosystems (Begon et al. 2006; Estes et al. 2011). In natural communities, species are embedded in multispecies food webs of consumer-resource interactions (McCann 2011). These interactions can be complex and lead to both direct and indirect effects due to regularly observed phenomena such as omnivory (Pimm and Lawton 1978; Kratina et al. 2012) and intraguild predation (Polis et al. 1989; Arim and Marquet, 2004). The combination of these direct and indirect effects are important ecologically (Strauss 1991; Wootton 1994; Miller and terHorst 2012), but can also have important evolutionary consequences (Miller and Travis 1996; Walsh and Reznick 2008; Walsh 2013; Patel and Schreiber 2015; terHorst 2010; terHorst et al. 2015), resulting in complex evolutionary 81 dynamics in multispecies communities.

82 How the complexity that arises from multiple species interactions affects the stability of those 83 communities has been the subject of debate in ecology for decades (Pimm 1991; McCann 2000). The 84 thought that diversity should stabilize communities, proposed by early ecologists (MacArthur 1955;

85 Elton 1958) was challenged by May (1974), who demonstrated mathematically that increases in 86 diversity result in a decreased likelihood of local stability in randomly interacting communities.

87 Analogous stability criteria have since been described for a variety of more realistic ecological 88 interaction structures (Allesina and Tang 2012). Ecologists have also investigated how trait variation 89 and evolution affect ecological stability. Kondoh (2003) found that predators who behaviorally alter 90 their foraging patterns to match an optimal niche increase species diversity and stabilize communities. 
91 This result echoes the early ideas that more diverse communities can be buffered through prey

92 switching (MacArthur 1955; Elton 1958).

93 Here, we move beyond plastic changes in traits, such as behavioral modification, to investigate

94 whether rapid trait evolution can have similar stabilizing effects in diverse ecological communities. We

95 are particularly interested in whether rapid trait evolution can confer stability to communities through

96 evolutionary rescue. While previous discussions of evolutionary rescue have focused on shifts in the

97 abiotic environment (e.g., Bell 2013), we are interested in scenarios where populations can be

98 evolutionarily rescued following changes in the community composition (Kovach-Orr and Fussman

99 2013; Yamamichi and Miner 2015). For example, prey that are driven to low densities by predators

100 may recover because of ecological feedbacks that lead to predator-prey oscillations. Alternatively, prey

101 populations may adapt in response to strong selection by predation pressure, and the surviving resistant

102 genotypes increase the prey population size.

103

104 We built a stochastic, individual-based simulation model to study how the ecological and evolutionary

105 effects of intraspecific trait variation influence stability in food web communities. We found that trait

106 evolution stabilized these food web communities by slowing the rate of species extinctions on short

107 time scales. On longer time scales, however, trait evolution had a less pronounced effect on the number

108 of extinctions. A key mechanism underlying this increase in this transient increase persistence is

109 population rescue via trait evolution. We did not find strong effects of species richness on the

110 evolutionary effect on food web stability. Further, we found that this evolutionary stabilizing effect on

111 communities relied predominantly upon the novel generation of genetic variation through mutation,

112 rather than selection on preexisting standing variation. 


\section{Models and Methods}

\section{The Eco-evolutionary model}

115 To study whether trait evolution confers stability to food web communities, we developed and

116 simulated the dynamics of a stochastic, individual-based eco-evolutionary quantitative genetics model.

117 The model consists of $N_{B}$ basal species and $N_{C}$ consumer species. Basal species are autotrophs (i.e., do

118 not need to consume other individuals in order to reproduce), and are assumed to have no intraspecific

119 trait variation, and thus do not evolve in our model. Consumer species, on the other hand, have

120 intraspecific trait variation, and can evolve over time. These species are omnivorous (i.e., can

121 potentially eat both basal and other consumer species), however, cannibalistic interactions are

122 forbidden. Inclusion of cannibalism is beyond the scope of our current study, but we recognize it's

123 potential importance for ecological communities (Fox 1975; Rudolf 2007). While only consumer

124 species are able to evolve in our model, both consumer and basal species exhibit ecological dynamics,

125 and can go extinct.

126 The per-capita birth rate of the $i$-th basal species is $b\left(1-R_{i} / S\right)$ where $b$ is the maximal per-capita birth

127 rate, $R_{i}$ is the density of the basal species, and $S$ is the maximal number of occupiable sites available to

128 species $i$. In the absence of predation, the per-capita death rates of all basal species are a constant $d$.

129 The attack rate of a consumer individual on a hetereospecific individual depends on the trait values of

130 the interacting individuals (Nuismer and Doebeli 2004; Nuismer et al. 2005; Schreiber et al. 2011;

131 Patel and Schreiber 2015). That is, an individual with trait value $y$ attacks an individual with trait value $132 x$ at the following rate $a(y, x)$ :

$$
\alpha e^{-\frac{(\mathrm{y}-\theta-\mathrm{x})^{2}}{2 \tau^{2}}}
$$


133 where $\alpha$ is the maximum possible attack rate, $\theta$ is the optimal difference in trait values that maximizes

134 the attack rate, and $\tau$ determines the degree of specialization of the predator (i.e., diet breadth) (fig.

$1351 A)$.

136 To describe the per-capita mortality rates due to consumption and the consumer birth rates, let

$137 y_{i 1}, y_{i 2}, \ldots, y_{i C_{i}}$ be the trait values of $C_{i}$ individuals of consumer species $i$. Then the per-capita mortality

138 rate due to consumption for an individual of consumer species $i$ with trait $y$ is

$$
M_{i}^{C}(y)=\sum_{\substack{j=1 \\ j \neq i}}^{N_{C}} \sum_{k=1}^{C_{j}} a\left(y, y_{j k}\right)
$$

139 The per-capita mortality rate of an individual of basal species $i$ is:

$$
M_{i}^{B}=\sum_{j=1}^{N_{C}} \sum_{k=1}^{C_{j}} a\left(x_{i}, y_{j k}\right)
$$

140 where $x_{i}$ is the trait value for all individuals of basal species $i$. The birth rate of an individual consumer

141 of species $i$ with trait $y$ is proportional to its consumption rate:

$$
B_{i}(y)=e \sum_{j=1}^{N_{R}} a\left(x_{j}, y\right) R_{j}+e \sum_{\substack{j=1 \\ j \neq i}}^{N_{C}} \sum_{k=1}^{C_{j}} a\left(y_{j k}, y\right)
$$

142 where the proportionality constant $e$ corresponds to the conversion efficiency of a consumer. The first

143 term of this sum corresponds to the consumption of basal species, while the second term corresponds

144 to the consumption of hetereospecific consumers. When a consumer individual reproduces, it

145 randomly mates with another conspecific individual. The trait value of the offspring is normally 
146 distributed around the mean of its parental trait values with mutational variance $\sigma^{2}$ (Slatkin 1970;

147 Lynch \& Walsh 1998) (fig. 1B). With higher $\sigma$ values, populations evolutionarily explore trait space

148 more rapidly.

149 The model corresponds to a continuous-time Markov chain (Norris 1998) where individuals are

150 updated at the previously described rates. That is, over a sufficiently small time interval $\Delta t$, the

151 probability of an individual of consumer species $i$ with trait value $y$ giving birth is (approximately)

$152 B_{i}(y) \Delta t$ and the probability of this individual dying is (approximately) $d \Delta t+M_{i}^{C}(y) \Delta t$. The

153 probability of an individual of basal species $i$ giving birth is (approximately) $b\left(1-R_{i} / K\right) \Delta t$ while the

154 probability of this individual dying is (approximately) $d \Delta t+M_{i}^{R} \Delta t$. The limit as $\Delta t$ goes to zero

155 determines the continuous-time Markov chain. As doing exact simulations with Gillespie's algorithm

156 (Gillespie 1977) is computationally expensive, we use the tau-leaping approach (Cao et al. 2005, 2006)

157 in which one performs simultaneous independent updates for sufficiently small $\Delta t$. This method has

158 been proven to approximate the distributional dynamics of the exact simulations (Anderson et al.

159 2011).

160 Methods

161 We ran continuous-time, stochastic, individual-based simulations of communities following the model

162 described above. In these simulations, we tracked changes in both population densities and mean trait

163 values through time. We varied the amount of trait variation in the communities to assess the effect of

164 rapid evolution in consumers. Finally, we ran alternative sets of simulations where trait variation is

165 either generated solely through mutation or solely through pre-existing standing variation. In all cases,

166 basal species trait values were fixed through time.

167 Treatments and replication. For both trait variation regimes, we simulated eco-evolutionary dynamics 
168 of 500 communities with $N_{T}=20$ (10 consumer and 10 basal species). In all cases, basal species had

169 birth rates $b=0.25$ and death rates $d=0.1$, with population carrying capacities of 200 individuals.

170 Consumer attack rate parameters were held constant for all consumer species: $\alpha=0.001, \tau=0.1$, and $\theta$

$171=1$. Consumer species converted consumed prey with efficiency $e=0.75$ and died at rate $\delta=0.01$.

172 Each simulation ran for 10,000 time steps. We scaled these time steps to the life expectancy for a

173 typical individual of a basal species, as $(1 / d \Delta \mathrm{t})$, resulting in approximately 250 basal species

174 generations per simulation. For each full set of $\sigma^{2}$ values $[0,1.5]$, the initial trait values for all species

175 were independent draws from a uniform distribution on the interval $[0,5]$. We tracked ecological

176 (changes in population densities) and evolutionary (changes in trait values) dynamics for each species

177 in the community (fig. 2). While we primarily focused on communities with total initial richness of $N_{T}$

$178=20$, we also simulated communities with total species richness ranging over $N_{T}=\{4,6,10,20\}$ (all

179 with equal numbers of basal $N_{B}$ and consumer species $\left.N_{C}\right)$ and $\sigma^{2}$ in $[0,1.5]$, with 50 replicates for each

180 treatment combination. Each community had initial trait values for the species (both basal and

181 consumer) drawn randomly from a uniform distribution on the interval [0,5]. We found little effect of

182 species richness on extinction probability, and no significant interaction with the effects of evolution.

183 These additional results are presented in Appendix A.

184 Trait variation regimes. To explore the effect of trait variation $\sigma^{2}$ on community dynamics, we

185 imposed two distinct regimes that determined how trait variation was generated. In the 'standing

variation' regime, we introduced standing trait variation $\sigma^{2}$ at the beginning of each simulation, and no

187 subsequent trait variation was generated through mutation (i.e., the trait of all offspring is the midpoint

188 of their parental traits). In the 'mutation only' regime, we initiated simulations with no initial trait

189 variation, but mutations contributed to trait variation through time. In this 'mutation only' regime, all

190 intraspecific trait variation arises via mutation over the course of the simulations. Comparison between 
191 these trait variation regimes allows us to determine whether lineage sorting of standing variation or

192 continual mutation and selection contributes more to evolutionary effects on community dynamics

193 (Appendix B).

194 Extinctions and species persistence. For both consumer and basal species, we measured stability

195 through species persistence, i.e., the fraction of species persisting throughout the simulations. We

196 measured both the rate of species losses as well as the total number of extinctions at the end of the

197 simulations. The simulation data allowed us not only to determine the number of species extinctions in

198 each community (extent of extinction), but also the timing of these extinction events (dynamics and

199 rate of extinctions). We then compared how trait evolution affects the extent and rate of species

200 extinction by looking at extinction patterns as a function of the mutational variance or initial standing

201 variation $\left(\sigma^{2}\right)$. We defined the extent of extinction as the proportion of species at the beginning of the

202 simulation that go extinct by the end of the simulation. To quantify the rate of extinction, we calculated

203 the accumulation of extinction events through time and the average time for a community to

204 experience $50 \%$ of its eventual extinction events.

205 Population rescues. To quantify the effect of trait evolution on consumer population rescues, we

206 counted the number of times a population crossed a threshold density $L$ (fig. 3). Each of these

207 categories (i.e., number of threshold crossings) has a distinct biological interpretation. If a population

208 never crossed the threshold, it means that the population persisted for the entire simulation while never

209 falling to low densities. ('Low' is defined by the threshold density). If a population crossed the

210 threshold density only once, we found that in our simulations the population always went extinct by

211 the end of our simulations. Two crossings of the threshold density indicated that the population fell to

212 low density, but was subsequently rescued and continued to persist to the end of the simulation. This

213 scenario captures the iconic U-shaped population trajectory of evolutionary rescue (Gomulkiewicz and 
214 Holt 1995). Three crossings indicated a population was rescued, but then suffered subsequent

215 extinction. More crossings ( $>3$ ) indicated multiple rescues from repeated low density, either with

216 eventual persistence (an even number of crossings) or extinction (an odd numbers of crossings). We

217 used a threshold density of $L=25$ individuals, approximately $10 \%$ of typical peak population densities.

218 We calculated the number of threshold crossings from smoothed population trajectory data to ignore

219 small, random changes in population densities using the 'filter' function in R. While we quantified the

220 number of threshold crossings, our presentation focuses on the number of rescues (the floor of half of

221 the number of threshold crossings), the biologically more relevant quantity. For each rescue event,

222 then, the population can either persist or subsequently go extinct.

223 Dynamics of trait variation. We examined the dynamics of trait variation with two methods. First, we

224 quantified the standard deviation of population trait values for all populations through time for both the 225 mutation only and standing variation regimes. This approach gave a general picture of differences in

226 how trait variation was generated and maintained in the simulated communities. Second, we

227 investigated whether the patterns of intraspecific trait variation correlated with population rescues. To

228 do so, we measured trait variation in populations at the times at which they crossed the low density

229 threshold $L$. That is, at the time when a population crossed $L$, we measured the standard deviation in its

230 trait values. All simulations and subsequent data analyses were written and performed in R (R Core

231 Development Team 2014).

\section{Results}

\section{Extinction and persistence}

234 The parameter controlling the rate of trait evolution $\left(\sigma^{2}\right)$ influenced the dynamics of population

235 extinctions from communities by changing both the rate and extent of species losses (fig. 4). Increasing 
$236 \sigma^{2}$ resulted in slowing of the consumer extinction rates (fig. $4 B, E$ ). We also tracked the basal

237 extinctions over our simulation (fig. $4 A, D$ ). We found that at low $\sigma^{2}$ values, basal extinctions are the

238 highest, with no extinctions occurring at $\sigma^{2}>0.5$. At low $\sigma^{2}$ values $(0.1-0.2)$, consumer populations

239 drove basal species extinct, and had the potential to evolve to utilize new basal species, resulting in the

240 continued accumulation of basal extinctions at these low levels of mutational variance (fig. 4B). This

241 decrease in basal extinctions at higher values of $\sigma^{2}$ can be attributed to the decreasing ability of a

242 population to efficiently exploit the basal species when consumer populations have more variable trait

243 values. As $\sigma^{2}$ increases, the average attack rate of each consumer individual decreases (fig. C1). This

244 decrease can be interpreted as a cost of trait variation (Schreiber et al. 2011).

245 As a result, we see that the number of basal species extinctions peaks at low values of $\sigma^{2}(0.1)$, then

246 quickly falls to zero as $\sigma^{2}$ increases (fig. 4D). In contrast, the extent of consumer extinctions is

247 relatively constant with respect to $\sigma^{2}$ (fig. $4 E$ ). Figure $4 D$ and $4 E$ show the shifting relationship

248 between $\sigma^{2}$ and the number of extinctions over time. The bottom and top curves in each panel represent

249 the extent of extinction at early and late time points, respectively in the simulations, across a range of

$250 \sigma^{2}$ values. For consumer species, we show that the effect of $\sigma^{2}$ on the extent of extinctions becomes less

251 negative over time (fig. $4 E$ ). However, for basal species extinctions continue to accumulate at lower

252 values of $\sigma^{2}$, leading to extinction peaks at these values of $\sigma^{2}$ (fig. $4 D$ ). This peak at $\sigma^{2}=0.1$

253 corresponds to the slight increase in the number of consumer extinctions, and is reflected in the

254 dynamics of total community extinction (fig. $4 F$ ).

\section{Population rescues}

256 For any given number of rescues, populations can either persist or eventually go extinct. We found that

257 the number of populations that undergo rescue $(2+$ crossings $)$ increases with $\sigma^{2}$, while the number of 
258 populations that directly go extinct (one threshold crossing) or persist at high density without falling to

259 low density (zero threshold crossings) decreases with $\sigma^{2}$ (fig. $5 A$ ).

\section{Trait variation regimes}

261 In simulations in which trait variation is generated via mutations, the proportion of populations that 262 were rescued one or multiple times increased with $\sigma^{2}$ (fig. $5 A$ ). By contrast, in simulations with only 263 standing variation, the proportion of populations that were rescued once increased with $\sigma^{2}$, but the 264 proportion of multiple rescues (which is close to zero) was relatively unaffected by $\sigma^{2}$ (fig. $5 B$ ).

265 Trait variation due to mutations or standing genetic variation differentially affected the proportion of 266 populations that persisted without falling to low density (zero threshold crossings) (fig. 5). With 267 mutational input, the proportion of these persisting populations decreased rapidly at low values of $\sigma^{2}$, 268 and then slightly increased at high values of $\sigma^{2}$. With standing variation only, the proportion of these 269 persisting populations decreased in a linear fashion with increasing $\sigma^{2}$.

Exhaustion of genetic variation

271 Mutations generated trait variation throughout the simulations as long as populations persisted, while 272 trait variation was exhausted quickly when initial standing variation was the only source of trait 273 variation (fig. 6A). Mutations generated trait variation to the same level of variation that existed at the 274 beginning of the standing variation regime within 100 time steps $(\sim 2.5$ basal species generations $)$ at the 275 beginning of the simulations. However, mutations continually renewed this variation, whereas without 276 mutations, the variation was rapidly lost.

277 At times of crossing the threshold density, the standard deviation of trait values was significantly 278 higher for the mutation only regime (fig. $6 B$ ). In particular, at the time of secondary rescue events $(4+$ 
279 threshold crossings), the trait variation in the standing variation only regime fell to virtually zero (fig.

$2806 C$ ). By contrast, the standard deviations in trait values for the 'mutation only' regime were

281 approximately equal for all secondary threshold crossings, with slightly lower values at the first

282 crossing. This suggests that before the first threshold crossing, at which point the population is

283 decreasing, mutations had not generated as much variation as later in the simulation. Indeed, with

284 standing variation, the mean time to the first rescue event was significantly lower than the mutation

285 only regime (Mann-Whitney $\mathrm{U}$ test, $p<0.0001$; fig. B1).

\section{Discussion}

Using individual-based, stochastic simulations of an eco-evolutionary model, we found that rapid trait evolution increases persistence by slowing the rate of species extinctions. The slower pace of extinctions found with increased mutational variance (i.e., evolutionary potential) is a result of evolutionary rescues from low population densities. While the rate of evolution - controlled by the mutational variance parameter $\sigma^{2}$ - does not have a major effect on the number of eventual consumer extinctions in the long term, increased evolution substantially decreases the rate of extinctions on shorter time scales.

In the absence of evolution, extinctions occur very rapidly until the community reaches a stable state determine which species go extinct. For instance, if the trait values of the initial community result in very low attack rates by a consumer species (no or few prey individuals available), then that population will starve and go extinct. Likewise, a consumer near the optimum on a basal species can rapidly drive 
consumer species' extinction.

302 At low values of $\sigma^{2}$, consumers are able to evolve to overexploit basal species and drive them extinct.

303 This overexploitation does not occur at higher mutational variances because the consumer attack rates

304 are spread out among more basal species, allowing each population to maintain growth despite some

305 consumption. Because there is no trait variation in basal species, increasing trait variation in consumer

306 populations means that more consumer individuals are further away from the optimal trait difference

307 from basal species $(\theta)$, thereby lowering average attack rates, and generating a fitness load on

308 consumers (fig. C1). The consumers driving basal species extinct leads to an increase in subsequent

309 consumer extinctions, resulting in a slight peak in the extent of consumer extinctions at $\sigma^{2}=0.1$ (fig.

$3104 E)$.

311 With trait variation, consumer species are able to evolve to consume new species if they drive one

312 extinct. This can particularly be seen in basal species (fig. $4 A$ ), where the accumulation of extinctions

313 reaches an approximate equilibrium quickly, while with trait variation, basal extinctions continue to

314 accumulate. An analogous, but less pronounced, pattern is seen in the accumulation of consumer

315 extensions (fig. 4B). However, as sigma values increase further, the load of trait variation suppresses

316 the ability of consumers to drive prey species extinct (either basal or other consumers). This conclusion

317 is further supported in the 'standing variation only' model, in which there is no mutational input, and

318 therefore across all values of $\sigma^{2}$, the curves for extinction accumulation level off more quickly (fig.

319 B1). In the case of standing variation only, however, the load of trait variation vanishes because the

320 variation is quickly lost (fig. 6).

321 Our results illustrate two ways that intraspecific trait variation can affect ecological communities. First,

322 trait variation provides an opportunity for evolutionary change. We show that with inputs of trait 
323 variation through time, populations are able to avoid extinction through evolutionary change, leading

324 to increased persistence. Second, beyond the opportunity for selection, trait variation can alter

325 ecological interactions strengths, as exemplified by the reduced consumer attack rates at higher values

326 of $\sigma^{2}$ (fig. C1). Our simulations show how these two consequences of intraspecific trait variation can

327 jointly affect community dynamics. This is most clearly demonstrated by the contrast in the number of

328 basal species extinctions at low and high values of mutational variance (fig. $4 A, D$ ). These basal

329 extinctions can then affect subsequent extinctions in the community. Populations with the same

330 population trait means can have starkly different interaction strengths, reflecting the fitness load of

331 increased $\sigma^{2}$ (fig. C1; Schreiber et al. 2011). In nature, there is natural variation in many traits

332 measured across taxonomic systems and environments (Lynch and Walsh 1998; Bolnick et al. 2011),

333 and as such, ecologists should consider how trait variation affects species interactions within

334 communities for more realistic predictions for outcomes of species interactions.

335 Introducing mutation and evolution into our simulations has different effects on species extinctions

336 over short and long time scales. On shorter time scales, increasing $\sigma^{2}$ stabilizes communities by

337 slowing extinctions, but has diminishing effect over longer time scales. This difference among time

338 scales of the effect of increasing $\sigma^{2}$ is best illustrated in figure $4 F$, where the early negative relationship

339 flattens over time. This suggests that early evolutionary and extinction dynamics result in a community

340 structure that is relatively stable in the long term.

341 Extinction and evolution can alter the ecological connectedness in these communities, resulting in

342 novel relationships among community members. In an individual-based population genetic model of

343 predator-prey communities, Yamaguchi et al. (2011) also found that evolution could affect the number

344 of extinctions in communities. In their two-trophic level system, they found that the effects of

345 evolution on extinctions were dependent on the connectivity and genetic architecture. Evolution 
346 allowed consumers to change their prey use (i.e., gain or loss of trophic links), which could increase

347 extinctions through altered competitive exclusion dynamics or decrease extinction via escape from

348 competition among predators (Yamaguchi et al. 2011). We also found that evolutionary losses and

349 gains of trophic links affected extinction dynamics, through overexploitation of prey species and

350 evolutionary rescue of declining consumer populations. However, unlike their model, our communities

351 were not strictly two trophic levels. In our model, because consumers can both evolve and eat each

352 other, dynamics of trophic interaction losses and gains are complex. Interestingly, Yamaguchi et al.

353 found that the specifics of the predator's genetic system dictated whether the loss or gain of trophic

354 links affected stability. Assessing the effects of variable genetic systems in our system is a compelling

355 topic for future study (Schreiber et al. 2016).

Mechanisms of rescue

358 Our method of quantifying rescue events as the number of threshold-crossings (fig. 4) does not provide

359 direct information for the mechanisms underlying the rescue events. In the most intuitive rescue

360 scenario, a focal population can be rescued from low density if the trait values of that focal species

361 change such that some individuals are able to survive and reproduce (e.g., evolve to utilize a new prey

362 resource). However, the mechanism of rescue may be indirect. For instance, a competing species could

363 go extinct, or trait evolution in other community members could indirectly allow the focal species to

364 rebound (e.g., Yamamichi and Miner 2015).

365 More simply, the multiple rescue events we found at higher $\sigma^{2}$ values could arise from ecological

366 dynamics in which predators abundances respond to prey abundances and vice versa, such as in

367 predator-prey cycles. In such cases, the density of the 'rescued' consumer population would dip below 
368 the threshold density and recover as the basal species recover. However, the difference between the

369 'mutation only' and 'standing variation' regimes suggest that this is not the case. If multiple population

370 rescues were driven by ecological dynamics, we would expect those dynamics to continue regardless

371 of the regime of trait variation. In other words, if rescue events were driven purely by these ecological

372 oscillations, we would not expect to see population rescues stop after the exhaustion of genetic

373 variation in the standing variation regime (fig. 5). Comparison of the relationship between number of

374 rescues and $\sigma^{2}$ across the trait variation regimes supports that trait evolution is leading to the rescue

375 events. This suggests that if populations are unable to continue to generate this intraspecific trait

376 variation, they may soon face extinction risk, as they will not be able to be rescued from subsequent

377 declines in density (fig. 6).

\section{Implications for conservation}

379 We find that evolution can substantially slow the rate of extinctions on short time scales, but that it 380 only modestly alters the extent of species loss from communities over longer periods of time. The short

381 time scales over which we observe these strong effects are on the order of $\sim 10$ basal species

382 generations, and thus relevant for most conservation efforts. The slowed extinction rate we found could

383 provide critical time necessary for secondary ecological or environmental processes to act. In

384 particular, this transient increase in persistence could be important when considering a broader

385 landscape perspective (Thompson 2005). In this study, we have only simulated eco-evolutionary

386 dynamics in a single focal community. We did not examine any effects of immigration or emigration

387 among communities. When considering broader meta-community dynamics (Holyoak et al. 2005;

388 Urban et al. 2008), the increased persistence though evolutionary rescue could have important effects

389 on the maintenance of diversity at a larger scale. The slowed rate of extinction allows for more

390 opportunity for rescue via immigration (Mouquet and Loreau 2002, 2003; Urban et al. 2008). Further, 
391 our model ignores other complexities that can alter eco-evolutionary outcomes, such as facilitative

392 interactions (Bruno et al. 2003) and spatiotemporal environmental variation (e.g., Levins 1968;

393 Mouquet and Loreau 2003).

394 Even with only standing genetic variation, we find that intraspecific trait variation is critical for 395 evolutionary rescue of declining populations in the short term. In fact, evolutionary rescues occur 396 significantly faster with standing variation (as opposed to waiting for relevant trait variation to 397 accumulate through mutation) (fig. B2). This points to the maintenance of genetic variation in species 398 of concern as an important aspect of conservation strategy (beyond the maintenance of variation for 399 inbreeding avoidance). This suggests that in species with relatively slow generation times - and 400 therefore reduced opportunity for rapid evolution on ecological timescales -- populations of these 401 species with low variation may need input of variation if they are to be rescued through evolutionary 402 mechanisms (Sgro et al. 2011; van Oppen et al. 2015; Whiteley et al. 2015).

\section{Future directions}

404 Relaxing several assumptions of the current work opens compelling avenues for future research. We 405 studied an abstract trait that governs ecological interactions among species in a food web community. 406 Future work should seek to assign more explicit biological identity to the trait values. For instance, we 407 could treat our trait values as body size, a variable trait that has been shown to determine feeding 408 relationships across a wide breath of taxa and communities (Brose et al. 2006). However, with more 409 explicit assignment of functional traits comes the attendant model complexities that would have to be 410 addressed, such as trait dependent demographic parameters (i.e., rates of metabolism, birth, and death; 411 Brose et al. 2006; Brose 2010).

412 Genetic rescue, as opposed to evolutionary rescue, can occur in declining populations by increasing 
413 fitness through the demographic contribution of immigrants. While such input of genetic variation can

414 facilitate declining populations (van Oppen et al. 2015; Whiteley et al. 2015), this will depend on the

415 genetic composition of the immigrants. In particular, when there is evolution in a focal/local

416 community, if the immigrants are arriving from a source community where the selection pressures are

417 not parallel to the local community, the effect of immigrants may have a variety of effects. It is

418 possible that they could allow the population to recover (if the immigrating genetic variants facilitate

419 response); alternatively, if the immigrants are phenotypically more ancestral, this could have the

420 opposite effect of genetic rescue in which the immigrants constrain the evolutionary change, thereby

421 increasing the likelihood of extinction. Further, immigration of basal species individuals could

422 potentially stabilize consumer species that otherwise overexploit the basal species. Such studies of eco423 evolutionary meta-community dynamics are compelling avenues for research (Urban et al. 2008; De

424 Meester et al. 2016; Wittmann and Fukami 2016).

\section{Conclusion}

426 We provide evidence that intraspecific trait variation and evolution significantly stabilize diverse 427 ecological food web communities. Our eco-evolutionary simulations revealed that, on short time 428 scales, the decreased extinction risk is a result of trait variation that allows species that have fallen to 429 low density to be rescued via evolutionary changes. These results contribute to our understanding of 430 how rapid evolution can generate patterns in ecological community dynamics that do not occur in the 431 absence of intraspecific variation. Moreover, the slowing of species losses from diverse communities 432 occurs on timescales relevant to conservation and management concerns, further stressing the value of 433 evaluating concurrent ecological and evolutionary processes. Our results support the calls for 434 accounting for intraspecific trait variation found in natural populations, and the resultant capacity for 435 trait variation and rapid evolution to affect important ecological phenomena. 


\section{Acknowledgements}

437 We thank Swati Patel and other members of the terHorst and Schreiber research groups for helpful 438 discussion. This work was funded by grants from the National Science Foundation to SJS and CPT 439 (DMS-1312490), to SJS (DMS-1313418), and to CPT (OCE-1559105).

\section{$441 \quad$ Literature Cited}

442 Allesina, S., and Tang, S. 2012. Stability criteria for complex ecosystems. Nature 483:205-208.

443 Arim, M., and Marquet, P.A. 2004. Intraguild predation: a widespread interaction related to species biology. Ecology Letters 7:557-564.

Anderson, D.F., Ganguly, A., and Kurtz, T.G. 2011. Error analysis of tau-leap simulation methods. Annals of Applied Probability 21:2226 - 2262.

Bassar, R.D., Marshall, M.C., López-Sepulcre, A., Zandonà, E., Auer, S.K., Travis, J., Pringle, C.M., Flecker, A.S., Thomas, S.A., Fraser, D.F., and Reznick, D.N. 2010. Local adaptation in trinidadian guppies alters ecosystem processes. Proceedings of the National Academy of Sciences 107:3616-3621.

Becks, L., Ellner, S.P., Jones, L.E., and Hairston, N.G. 2012. The functional genomics of an ecoevolutionary feedback loop: linking gene expression, trait evolution, and community dynamics. Ecology Letters 15:492-501.

Begon, M., Townsend, C.R., and Harper, J.L. 2006. Ecology: from individuals to ecosystems. Blackwell Publishers, Hoboken. 
Bolnick, D.I., Amarasekare, P., Araújo, M.S., Bü̈ger, R., Levine, J.M., Novak, M., Rudolf, V.H., Schreiber, S.J., Urban, M.C., and Vasseur, D.A. 2011. Why intraspecific trait variation matters in community ecology. Trends in Ecology and Evolution 26:183-192.

Brose, U. 2010. Body-mass constraints on foraging behaviour determine population and food-web dynamics. Functional Ecology 24: 28-34.

Brose, U., Jonsson, T., Berlow, E.L., Warren, P., Banasek-Richter, C., Bersier, L.F., Blanchard, J.L., Brey, T., Carpenter, S.R., Blandenier, M-F.C., Cushing, L., Dawah, H.A., Dell, T., Edwards, F., Harper-Smith, S., Jacob, U., Ledger, M.E., Martinez, N.D., Memmott, J., Mintenbeck, K., Pinnegar, J.K., Rall., B.C., Rayner, T.S., Reuman, D.C., Ruess, L., Ulrich, W., Williams, R.J., Woodward, G., and Cohen., J.E. 2006. Consumer-resource body-size relationships in natural food webs. Ecology 87:2411-2417.

Bruno, J.F., Stachowicz, J.J., and Bertness, M.D. 2003. Inclusion of facilitation into ecological theory. Trends in Ecology and Evolution 18:119-125.

Cao, Y., Gillespie, D.T., and Petzold, L.R. 2005. Avoiding negative populations in explicit Poisson tau-leaping. The Journal of Chemical Physics 123:054104.

Cao, Yang, Gillespie, D.T., and Petzold, L.R.Efficient step size selection for the tau-leaping simulation method. The Journal of Chemical Physics 124:044109.

Carlson, S.M., Cunningham, C.J., and Westley, P.A. 2014. Evolutionary rescue in a changing world. Trends in Ecology and Evolution 29:521-530.

De Meester, L., Vanoverbeke, J., Kilsdonk, L. J., and Urban, M. C. 2016. Evolving perspectives on monopolization and priority effects. Trends in Ecology and Evolution 31:136-146. 
Elton, C.S. 1958. The ecology of invasions by plants and animals. Methuen, London.

478 Estes, J.A., Terborgh, J., Brashares, J.S., Power, M.E., Berger, J., Bond, W.J., Carpenter, S.R., Essington,T.E., Holt, R.D., Jackson, J.B.C, Marquis, R.J., Oksanen, L., Oksanen, T., Paine, planet earth. Science 333:301-306.

Fox, L.R. 1975. Cannibalism in natural populations. Annual Review of Ecology and Systematics 6:87106.

Gomulkiewicz, R. and Holt, R.D. 1995. When does evolution by natural selection prevent extinction? Evolution 49:201-207.

Gonzalez, A., Ronce, O., Ferriere, R., and Hochberg, M.E. 2013. Evolutionary rescue: an emerging focus at the intersection between ecology and evolution. Philosophical Transactions of the Royal Society B: Biological Sciences 368:20120404.

Hairston, N.G., Ellner, S.P., Geber, M.A., Yoshida, T., and Fox, J.A. 2005. Rapid evolution and the convergence of ecological and evolutionary time. Ecology Letters 8:1114-1127.

495 Hendry, A. P., and Kinnison, M. T. 1999. Perspective: the pace of modern life: measuring rates of contemporary microevolution. Evolution 53:1637-1653. 
Hiltunen, T., Ellner, S.P., Hooker, G., Jones, L.E., and Hairston, N. 2014. Eco-evolutionary dynamics in a three-species food web with intraguild predation: intriguingly complex. Advances in Ecological Research 50:41-74.

Holyoak, M., Leibold, M.A., and Holt, R.D. 2005. Metacommunities: spatial dynamics and ecological communities. University of Chicago Press, Chicago.

Kondoh, M. 2003. Foraging adaptation and the relationship between food-web complexity and stability. Science 299:1388-1391.

Kovach-Orr, C., and Fussmann, G. F. 2013. Evolutionary and plastic rescue in multitrophic model communities. Philosophical Transactions of the Royal Society of London B: Biological Sciences 368:20120084.

Levins, R. 1968. Evolution in changing environments: some theoretical explorations. Princeton

Kratina, P., LeCraw, R.M., Ingram, T., and Anholt, B.R. 2012. Stability and persistence of food webs with omnivory: Is there a general pattern? Ecosphere 3:1-18.

511 Lynch, M., and Lande, R. 1993. Evolution and extinction in response to environmental change. Biotic interactions and global change. Pages 234-250 in Kareiva, P.M., and Kingsolver, J.G., eds. Biotic interactions and global change. Sinauer, Sunderland.

514 Lynch, M., and Walsh, B. 1998. Genetics and analysis of quantitative traits. Sinauer Sunderland.

515 MacArthur, R. 1955. Fluctuations of animal populations and a measure of community stability. 
517 May, R. 1974. Stability and complexity in model ecosystems. Princeton University Press, Princeton.

518 McCann, K.S. 2000. The diversity-stability debate. Nature 405:228-233.

519 McCann, K.S. 2011. Food webs. Princeton University Press, Princeton.

520 Miller, T.E., and terHorst, C.P. 2012. Indirect effects in communities and ecosystems. Oxford 521 Bibliographies. Oxford University Press, Oxford.

522 Miller, T. E., and Travis, J. 1996. The evolutionary role of indirect effects in $523 \quad$ communities. Ecology 77:1329-1335.

524 Miner, B.E., De Meester, L., Pfrender, M.E., Lampert, W., and Hairston, N.G. 2012. Linking genes to communities and ecosystems: Daphnia as an ecogenomic model. Proceedings of the Royal Society of London B: Biological Sciences 279:1873-1882.

Mouquet, N., and Loreau, M. 2002. Coexistence in metacommunities: the regional similarity hypothesis. The American Naturalist 159:420-426.

Mouquet, N., and Loreau, M. 2003. Community patterns in source-sink metacommunities. The American Naturalist 162:544-557.

531 Norris, J.R. 1998. Markov chains. Cambridge University Press, Cambridge.

532 Nuismer, S.L., and Doebeli, M. 2004. Genetic correlations and the coevolutionary dynamics of threespecies systems. Evolution 58:1165-1177.

534 Nuismer, S. L., Doebeli, M., and Browning, D. 2005. The coevolutionary dynamics of antagonistic interactions mediated by quantitative traits with evolving variances. Evolution 59:2073-2082.

536 Orr, H.A., and Unckless, R.L. 2014. The population genetics of evolutionary rescue. PLoS Genetics, 10:e1004551. 
540 Pantel, J. H., Duvivier, C., and Meester, L. D. 2015. Rapid local adaptation mediates zooplankton community assembly in experimental mesocosms. Ecology Letters 18:992-1000.

542 Pimm, S.L. 1991. The balance of nature? Ecological issues in the conservation of species and communities. University of Chicago Press, Chicago.

544 Pimm, S.L., and Lawton, J. 1978. On feeding on more than one trophic level. Nature 275:542-544.

545 Polis, G.A., Myers, C.A., and Holt, R.D. 1989. The ecology and evolution of intraguild predation: potential competitors that eat each other. Annual Review of Ecology and Systematics 20:297330.

Post, D.M., and Palkovacs, E.P. 2009. Eco-evolutionary feedbacks in community and ecosystem ecology: interactions between the ecological theatre and the evolutionary play. Philosophical Transactions of the Royal Society B: Biological Sciences 364:1629-1640.

Reznick, D. N., and Ghalambor, C. K. 2001. The population ecology of contemporary adaptations: what empirical studies reveal about the conditions that promote adaptive evolution. Genetica 112:183-198. variation within a predator population. Ecology 92:1582-1593. 
560 Schreiber, S.J., and Patel, S. 2015. Evolutionarily induced alternative states and coexistence in systems with apparent competition. Natural Resource Modeling 28:475-496.

562 Schreiber, S. J., Patel, S., and terHorst, C. 2016. Evolution as a coexistence mechanism: Does genetic architecture matter? arXiv preprint:1609.05571.

Sgro, C. M., Lowe, A. J., and Hoffmann, A. A. 2011. Building evolutionary resilience for conserving biodiversity under climate change. Evolutionary Applications 42:326-337.

567 Slatkin, M. 1970. Selection and polygenic characters. Proceedings of the National Academy of Sciences 66:87-93.

terHorst, C.P. 2010. Evolution in response to direct and indirect ecological effects in pitcher plant Strauss, S.Y. 1991. Indirect effects in community ecology: their definition, study and importance. inquiline communities. The American Naturalist 176:675-685.

terHorst, C.P., Lennon, J.T., and Lau, J.A. 2014. The relative importance of rapid evolution for plantmicrobe interactions depends on ecological context. Proceedings of the Royal Society B:

terHorst, C.P., Lau, J.A., Cooper, I.A., Keller, K.R., Rosa, R.J.L., Royer, A.M., Schultheis, E.H., Suwa, T., and Conner, J.K. 2015. Quantifying nonadditive selection caused by indirect Biological Sciences 281:20140028. effect size of predators in experimental microcosms. Ecology 91:629-636. 
581 Thompson, J.N. 2005. The geographic mosaic of coevolution. University of Chicago Press, Chicago.

582 Turcotte, M. M., Reznick, D. N., and Hare, J. D. 2011. The impact of rapid evolution on population dynamics in the wild: experimental test of eco-evolutionary dynamics. Ecology

van Oppen, M. J., Oliver, J. K., Putnam, H. M., and Gates, R. D. 2015. Building coral reef resilience Letters 14:1084-1092.

Urban, M.C., Leibold, M.A., Amarasekare, P., De Meester, L., Gomulkiewicz, R., Hochberg, M.E., Klausmeier, C.A., Loeuille, N., De Mazancourt, C., Norberg, J., Pantel, J.H., Strauss, S.Y., Vellend, M., and Wade, M.J. 2008. The evolutionary ecology of metacommunities. Trends in Ecology and Evolution 23:311-317.

Walsh, M.R. 2013. The evolutionary consequences of indirect effects. Trends in Ecology and through assisted evolution. Proceedings of the National Academy of Sciences 1128:2307-2313.

Vasseur, D.A., Amarasekare, P., Rudolf, V.H., and Levine, J.M. 2011. Eco-evolutionary dynamics enable coexistence via neighbor-dependent selection. The American Naturalist 178:E96-E109. Evolution 28:23-29.

Whiteley, A.R., Fitzpatrick, S.W., Funk, W.C., and Tallmon, D.A. 2015. Genetic rescue to the rescue.

Walsh, M.R., and Reznick, D.N. 2008. Interactions between the direct and indirect effects of predators determine life history evolution in a killifish. Proceedings of the National Academy of Sciences 105:594-599.

600 Trends in Ecology and Evolution 30:42-49. coexistence of species engaged in local priority effects. bioRxiv: 040659. 
602 Wootton, J.T. 1994. The nature and consequences of indirect effects in ecological communities. Annual Review of Ecology and Systematics 25:443-466.

604 Yamaguchi, W., Kondoh, M, and Kawata, M. 2011. Effects of evolutionary changes in prey use on the relationship between food web complexity and stability. Population Ecology 53:59-72.

606 Yamamichi, M., and Miner, B.E. 2015. Indirect evolutionary rescue: prey adapts, predator avoids extinction. Evolutionary Applications 8:787-795. 
624 Figure 1: Attack rate and reproduction. (A) Attack rate function. (B) Trait values of consumer

625 offspring are given by the deviation from the midparent mean trait value determined by the mutational

626 variance $\sigma^{2}$.

627
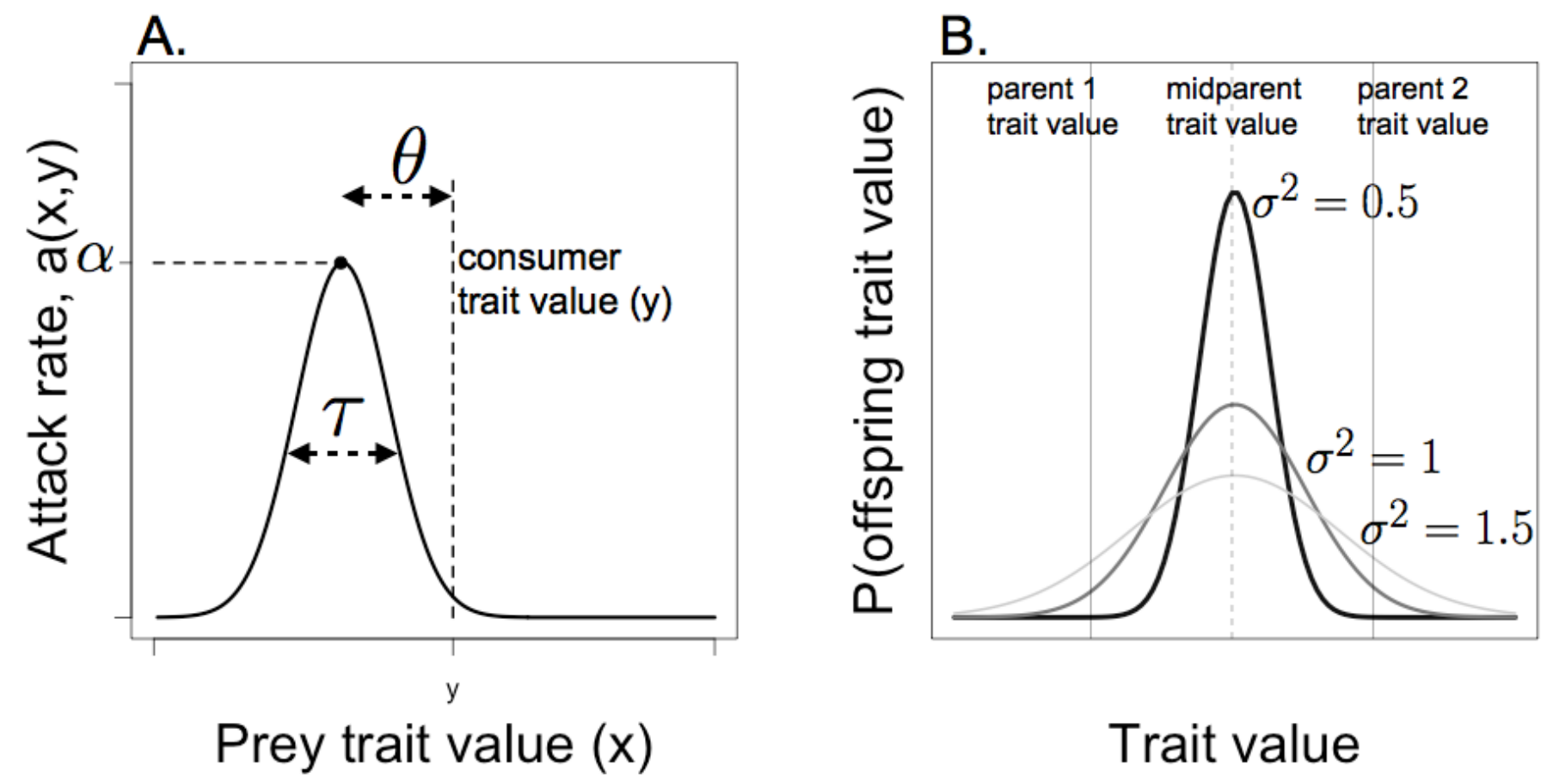
638 Figure 2: Representative ecological and evolutionary dynamics. (A) Ecological dynamics of basal

639 species. (B) Ecological dynamics of consumer species. (C) Consumer trait evolution dynamics. Each

640 curve shows dynamics for one consumer species. For the simulation shown, $\sigma^{2}=0.2$. Note that time

641 (x-axis) is on a log-scale.

642

643
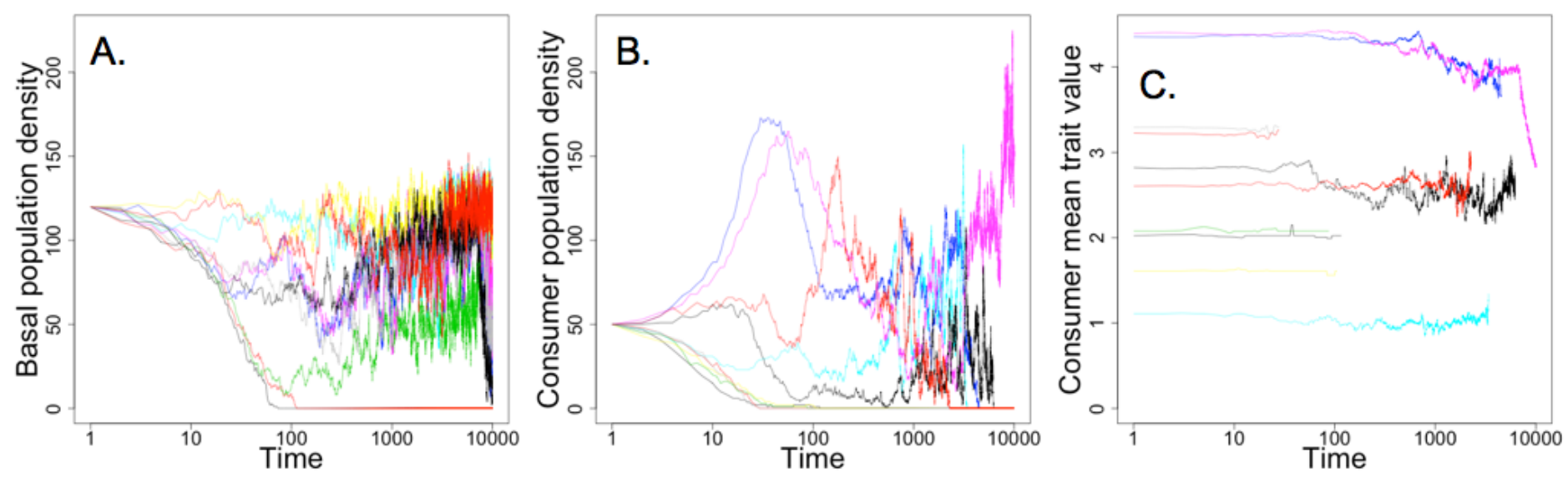
656 Figure 3: Classification of population rescues. Dashed line at $L$ shows the low density threshold for

657 population rescue. Each panel shows a classification of population rescues, with either eventual

658 persistence (dark line) or extinction (light line). (A) No rescues. (B) One rescue. (C) More than one

659 rescue.

660

661
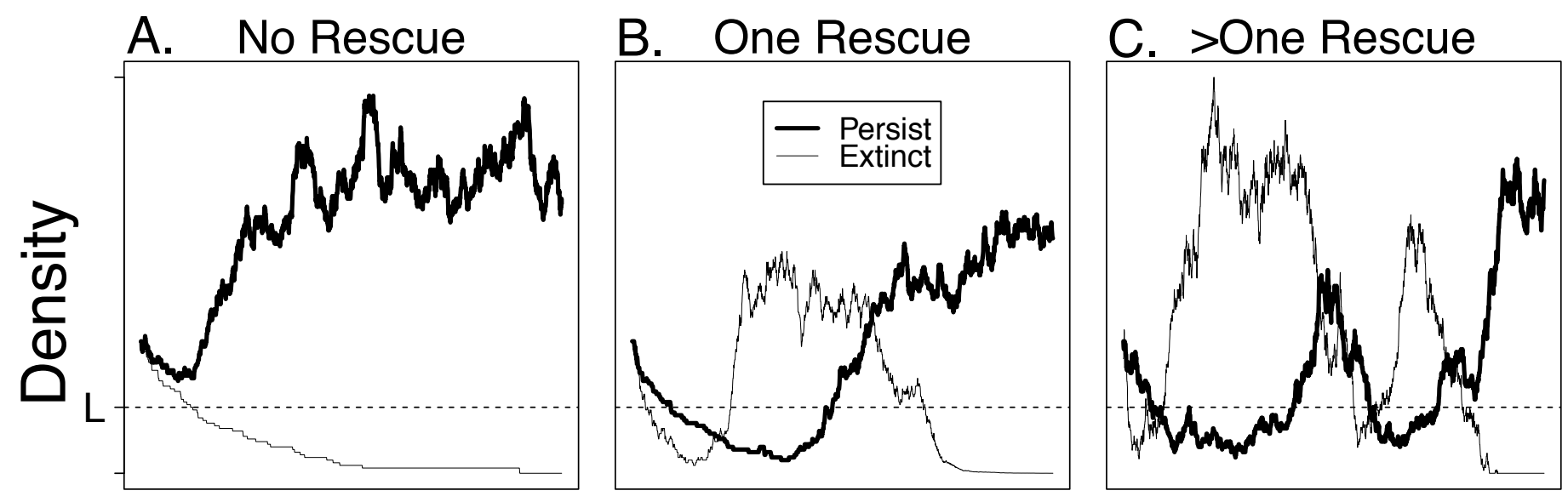

662

Time

663

664

665

666

667

668

669

670

671

672 
673 Figure 4: Extinction dynamics. (A-C) Each curve represents the accumulation of extinctions over time

674 for different values of the mutational variance $\sigma^{2}$. (D-F) The relationship between basal (B), consumer

675 (C), or species (D) extinctions and $\sigma^{2}$ at different points in time. The uppermost curves in (B)-(D) show

676 the mean number of extinctions at the end of our simulation runs. In all panels, colors represent

677 different values of $\sigma^{2}$.

678

679

680

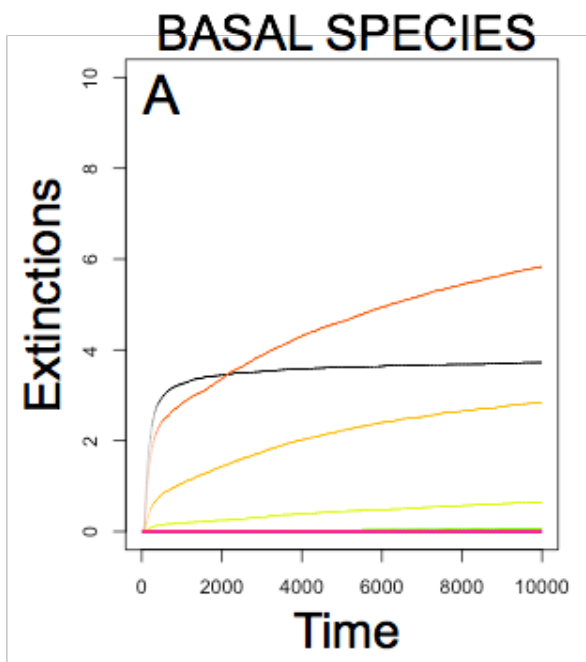

CONSUMER SPECIES
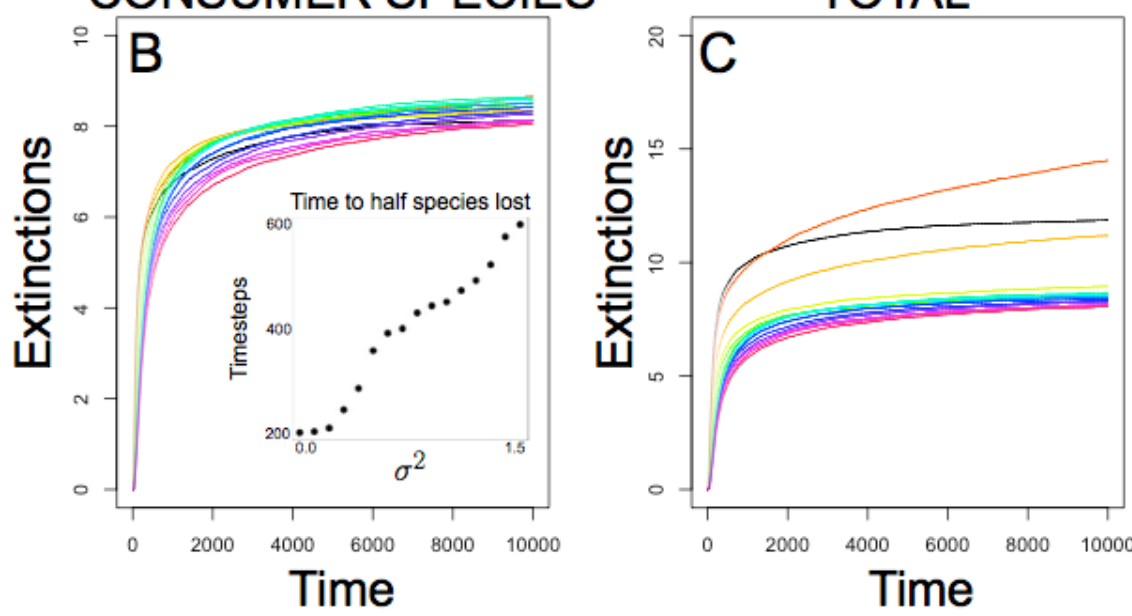

TOTAL
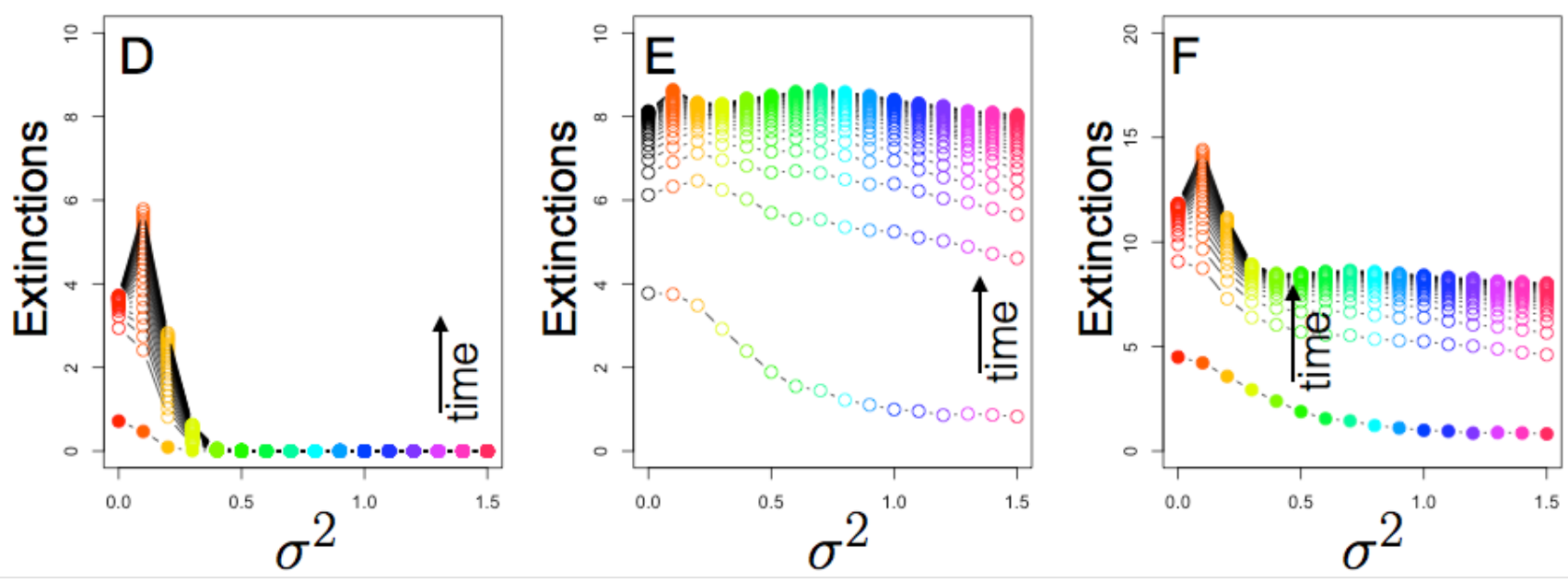

681

682 
683 Figure 5: Evolution increases proportion of populations rescued. (A) Evolutionary effect on rescue

684 under the 'mutation only' trait variation regime. Increased mutational variance $\left(\sigma^{2}\right)$ leads to more

685 trajectories with rescues (and multiple rescues). (B) 'Standing variation only' trait variation regime.

686 Here, probability of a trajectory having multiple rescues is not elevated. In both panels, the black

687 points show a decrease in the populations that have zero crossing, i.e., persist at high density. In all

688 cases, closed black circles represent the proportion. Upper panels (open black circles) in all cases show

689 the proportion of populations that directly go extinct (one crossing). Note that the y-axis scales are

690 different for lower and upper panels.

691

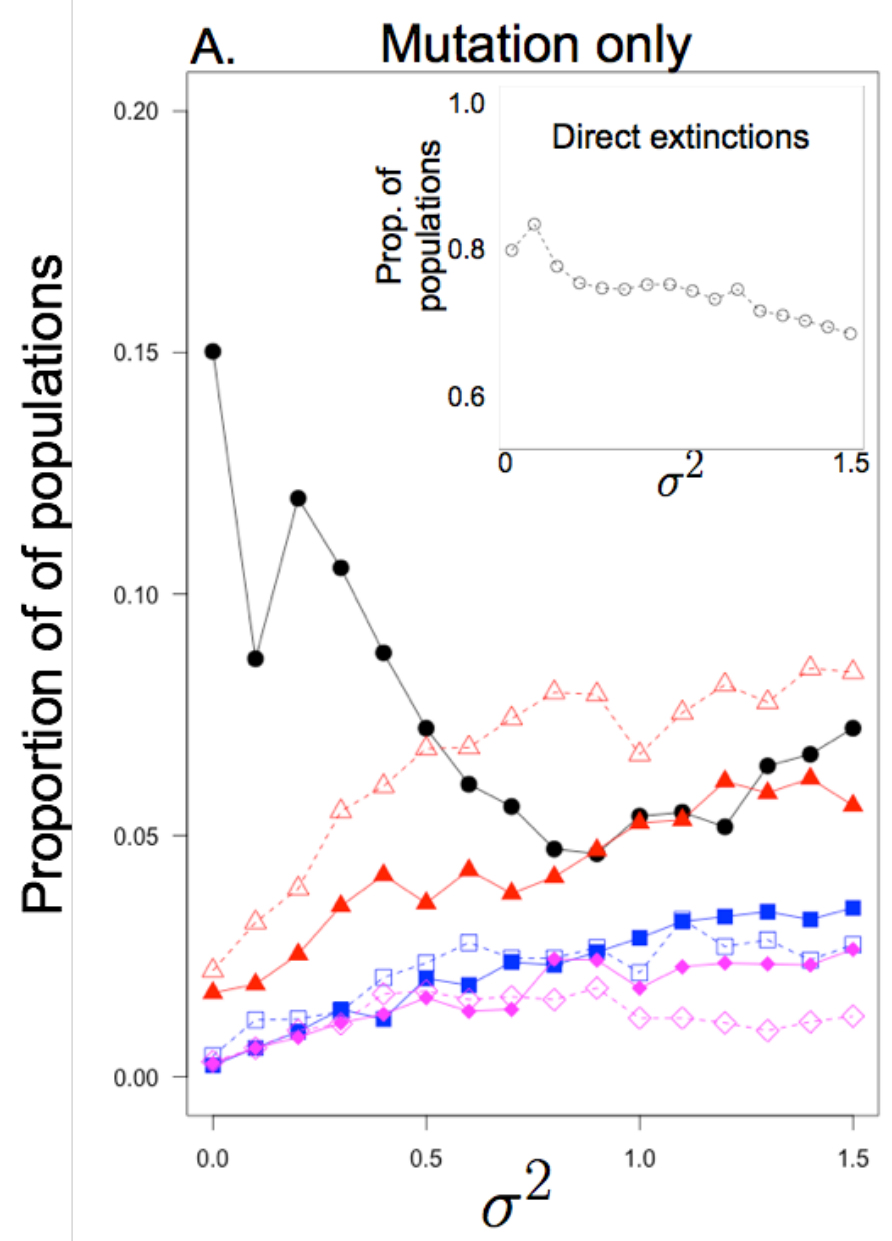

\section{B. Standing variation only}

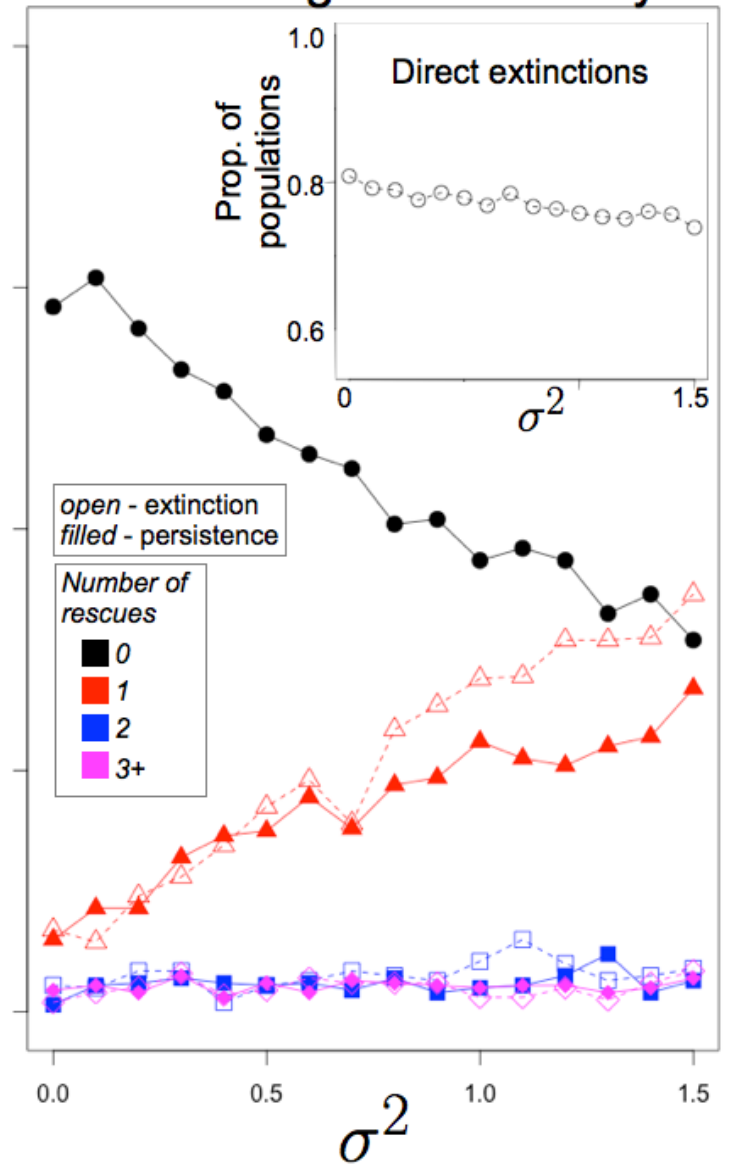


693 Figure 6: Standing variation only regime exhausts trait variation more quickly than mutation only

694 regime. (A) The mean standard deviation of the trait values decreases to zero in the standing variation

695 only regime, but stays level for the mutation only regime. Lower and upper bounds of shaded areas

696 represent the first and third quartiles, respectively. (B) At the time of threshold crossings, there is

697 significantly less intraspecific trait variation in the standing variation only regime (light) compared to

698 the mutation regime (dark). Arrows at top represent whether the populations are increasing or

699 decreasing in density at the time of threshold crossing. (C) Dark (mutation only) and light (standing

700 variation only) bars represent the total number of populations for each regime with each crossing. Note

701 the $y$-axis of $(C)$ is on logarithmic scale.

702
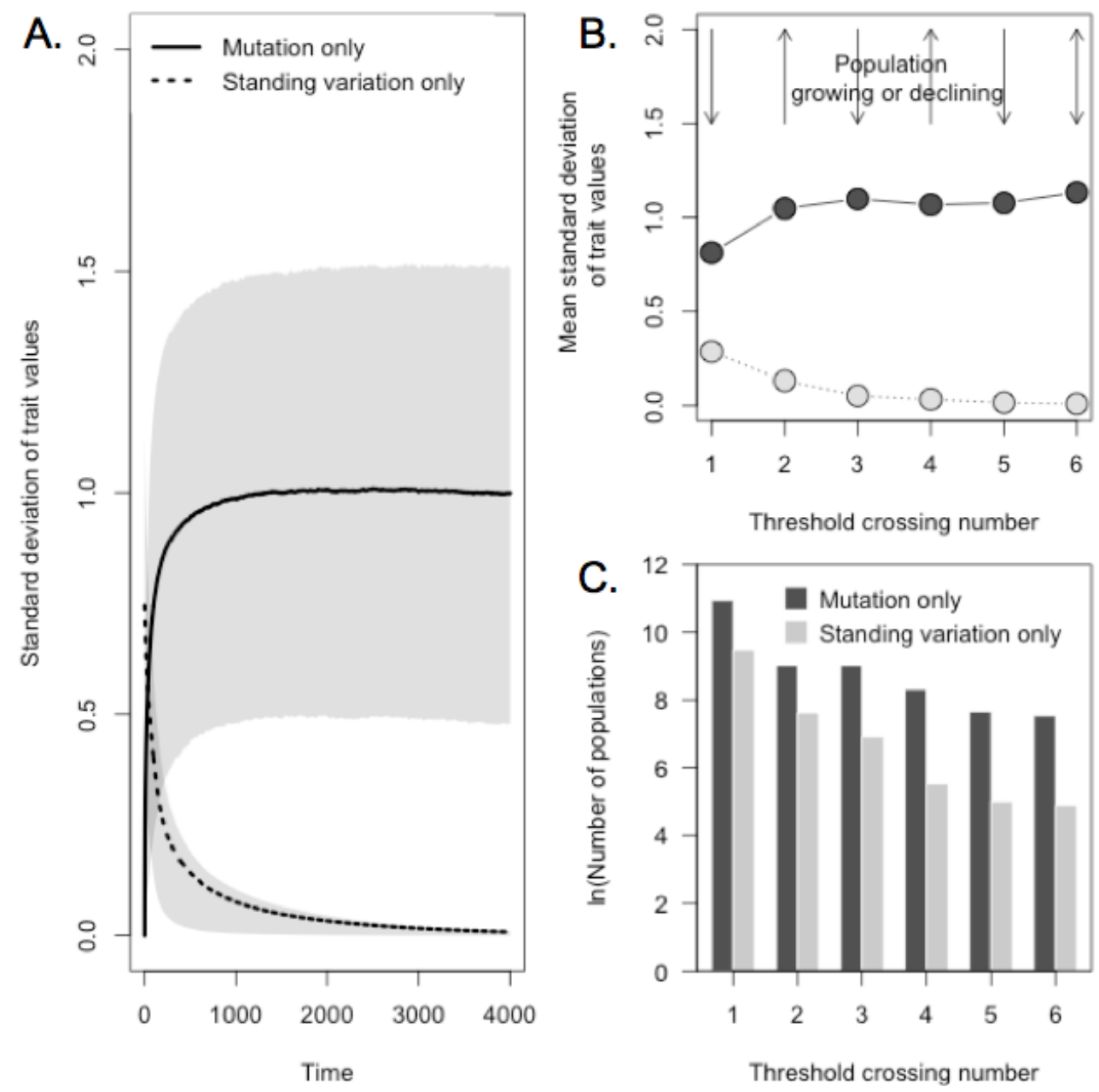

Threshold crossing number 
bioRxiv preprint doi: https://doi.org/10.1101/107763; this version posted February 11, 2017. The copyright holder for this preprint (which was not certified by peer review) is the author/funder, who has granted bioRxiv a license to display the preprint in perpetuity. It is made available under aCC-BY-NC-ND 4.0 International license. 